\title{
La reforma educativa de 2013, en el nivel de primaria. Discordancias entre la normatividad y el trabajo cotidiano del director
}

The educational reform of 2013, at the primary level. Discrepancies between the norms and the daily work of the director

\author{
David Castro Porcayo \\ e-mail: 1lanero68@live.com.mx \\ Universidad Nacional Autónoma de México. México
}

\section{Resumen}

El objetivo de este artículo es analizar la coherencia de la normatividad y la práctica de la gestión escolar en el nivel de primaria en México, con motivo de la aplicación de la reforma educativa del año 2013. La articulación de leyes y normatividades se encuentra en un proceso de integración, por lo que aún requiere ser analizado el proceso para su mejor aplicación en la vida cotidiana. Por tal situación, es posible que actualmente los directores se encuentren laborando bajo sistemas legales, administrativos y sindicales de tipo burocrático, pero deban presentar una evaluación de desempeño de acuerdo con perfiles, parámetros e indicadores que tienen un carácter "ideal" y que no concuerdan con la práctica profesional cotidiana.

Palabra clave: liderazgo; gestión escolar; director; evaluación; desempeño.

\begin{abstract}
The purpose of this article is to analyze the coherence of the normativity and the practice of school management at the primary level in Mexico, due to the application of the educational reform of 2013. The articulation of laws and regulations is found in an integration process, so the process still needs to be analyzed for its best application in everyday life. Due to this situation, it is possible that currently the directors are working under legal, administrative and union systems of bureaucratic type, but they must present a performance evaluation according to profiles, parameters and indicators that have an "ideal" character and that do not agree with daily professional practice.
\end{abstract}

Keywords: leadership; school management; principal; assessment; performance.

Recibido / Received: 31-10-2017

Aceptado / Accepted: 22-02-2018

Cómo referenciar este artículo / How to reference this article:

Castro, D. (2018). La reforma educativa de 2013, en el nivel de primaria. Discordancias entre la normatividad y el trabajo cotidiano del director. Tendencias Pedagógicas, 32, 119-132. doi: 10.15366/ tp2018.32.009 


\section{Introducción}

La modernización educativa, en México, ha tenido el propósito de cambiar la forma en que se está llevando a cabo la administración pública educativa. Tal proceso, en principio, se enfocó en la reordenación institucional, sindical, administrativa y financiera, además de la reformulación de contenidos y materiales educativos, la revaloración social de la función magisterial y la intención de fomentar una activa participación ciudadana. Posteriormente, se ha enfocado en el perfil y funciones de docentes y directivos.

Por un lado, a partir de corrientes teóricas como la Nueva Gerencia Pública (NGP) y eficacia escolar, se han identificado algunas características idóneas para mejorar la función directiva. Se pretende pasar de un director burocrático que solo aplica la normatividad a un gerente escolar proactivo, que domine tanto aspectos administrativos, como técnico-pedagógicos y que su liderazgo abarque, tanto el espacio escolar, como el comunitario. Por otro lado, en algunos estudios sobre la gestión escolar que realizó la Organización para la Cooperación y Desarrollo Económicos (OCDE) en México, halló, entre otros aspectos, que su actividad estaba caracterizada por actividades de tipo burocrático y el liderazgo era una faceta que requería ser fortalecida. Consecuentemente, con la reforma educativa del año 2013, la Secretaría de Educación Pública (SEP) llevó a cabo una revisión de los perfiles de los directivos, con motivo de la evaluación para el ingreso al Servicio Profesional Docente (SPD). La SEP destacó la importancia de contar con cuadros directivos que no sólo cumplan con tareas burocráticas, sino que apliquen principios de gestión educativa de forma eficaz y eficiente, y se tornen en líderes escolares.

La función directiva tomó importancia en las últimas décadas y ha llevado a plantear una reflexión sobre el perfil deseable del director, ya que sus funciones deben responder los retos que enfrentan las escuelas mexicanas dentro de un contexto de alta competencia internacional. En este esfuerzo, la articulación de leyes y normatividades se encuentra en un proceso de integración. Por lo que, es necesario revisar la coherencia entre las normas, por un lado, y su relación con la vida cotidiana de los directivos, por otro, para finalmente, ver si se integra a la práctica cotidiana. Algunas preguntas que se pueden generar de tal situación son las siguientes: ¿qué tipo de acciones ha implementado la Secretaría de Educación Pública para fortalecer el perfil del directivo?, ¿cómo se está presentando la articulación entre leyes, normas, evaluación y la vida cotidiana del director? Así, en el presente documento, en primer lugar, se establece un marco teórico y contextual, en segundo lugar, se describen algunas discordancias encontradas en la normatividad y el trabajo cotidiano del director para finalmente hacer una serie de observaciones.

\section{Marco teórico}

Pollit y Bouckaert (2000) consideran que, la Nueva Gerencia Pública, se entiende como la aplicación de tecnología de la gestión privada en las instituciones públicas; lo que ha implicado, la racionalización de los recursos, la reestructuración de las organizaciones y sus procesos, la revisión al proceso para la toma de decisiones y el incremento de la productividad de los empleados públicos. Además de una serie de cambios intencionales en las estructuras y procesos de organización del sector público. Se procura una actividad gubernamental que brinde servicios de calidad de forma económica y eficiente. Estos investigadores, resaltan la importancia de establecer un sistema de control político, mayor libertad a los administradores para llevar a cabo su función, transparencia en los procesos y mejorar la imagen de los funcionarios.

De acuerdo con Losada (1999), la Nueva Gestión Pública tiene cuatro características:

- La delegación de la toma de decisiones en instancias inferiores, ya que éstos están más próximos al problema y tienen objetivos más claros.

- Una orientación de sistemas de calidad en el proceso (insumos-proceso-producto).

- Trabajo enfocado en los usuarios del servicio (satisfacción del cliente).

- Una mayor orientación hacia el mercado, a través de contratos de gestión y personal, la 
competencia entre los organismos públicos, el cobro entre los organismos y recompensas orientadas en el desempeño.

Esta forma de comprender el funcionamiento de las organizaciones lucrativas ha permeado el ámbito educativo desde sus instituciones rectoras, hasta las escuelas. Básicamente, una Escuela efectiva se caracteriza por su capacidad para incrementar y agregar valor extra al aprendizaje de los alumnos. (Mortimore, 1991). Aunado a un enfoque de Escuelas eficaces, pretende identificar las características de las organizaciones escolares que obtienen mejores resultados, en cuanto a la forma, en que sus directivos, manejan sus recursos administrativos, humanos, financieros, contables o materiales.

Pozner (1997) incorpora la dimensión pedagógica a la gestión escolar y considera que es el conjunto de acciones relacionadas entre sí, que debe desarrollar un equipo directivo de una escuela para promover y posibilitar la consecución de la intencionalidad pedagógica en, con y para la comunidad educativa. Lo que implica considerar aspectos relacionados con la infraestructura, trabajo colegiado, formación y actualización de los docentes, control de procesos administrativos, vinculación con la comunidad, aspectos económicos o financieros, los cuales deben ser integrados de tal forma, que incidan en el logro de aprendizajes con calidad.

Desde hace algunas décadas, con esta perspectiva, algunos investigadores se han enfocado en determinar la importancia de la gestión directiva como un elemento clave para incrementar la calidad del servicio educativo y consecuentemente, el proceso de enseñanza-aprendizaje. (Branch, Hanushek y Rivkin, 2013). Se asume que las funciones directivas no podrían realizarse sin tener en cuenta el liderazgo. Existen diferentes tipos que pueden aplicarse al ámbito escolar, entre los que destacan: el transformacional, el distributivo y el comunitario. El liderazgo transformacional, según Burns (1978), refiere al tipo de líder que motiva a sus colaboradores para alcanzar un mejor rendimiento, a partir del intercambio de recompensas y, a partir de sus intervenciones, hacen que todo el trabajo escolar fluya sin problemas. Construye redes de trabajo que partan de la consideración de los miembros de forma individual, de la estimulación intelectual y proyección institucional (Álvarez y Pérez, 2011, p. 37). Las redes de colaboración y apoyo internas, se combinan con las externas para conseguir un intercambio de experiencias que benefician el trabajo cotidiano. El liderazgo distributivo, a decir de Leithwood y Riehl (2003 en Pont, Nusche y Moorman, 2008), es más una función que un puesto. Se asume que el liderazgo no debe confinarse jerárquicamente a funciones formales o posicionales, sino a una actividad que ejerce influencia en el grupo y que puede realizar cualquiera en cualquier nivel de la organización escolar. Esta forma de concebir el liderazgo permite involucrar a todos los miembros en la realización de un proyecto de manera corresponsable. Por su parte, el liderazgo comunitario es el trabajo que, como su nombre lo indica, realizan los directores, en el ámbito comunitario, los debe llevar a establecer relaciones de colaboración al interior de la comunidad escolar y con diversos actores del contexto local en el que se ubica la escuela. Esta forma de concebir el liderazgo permite involucrar a todos los miembros en la realización de un proyecto de manera corresponsable. Consecuentemente produce un sentido de pertenencia y conecta la voluntad de los miembros de una comunidad con una causa común que, en este caso, es elevar la calidad educativa. (Leithwood, Tomlinson y Genge, 1996).

Si se integran aspectos de liderazgo y gestión pública, se puede obtener un modelo como el que propone Leithwood. Este investigador considera ocho dimensiones de liderazgo y cuatro de gestión:

“1. Construir una visión de la escuela. A falta de una visión de la escuela, que aglutine los esfuerzos de cambio, no habrá una línea común de mejora. El liderazgo, en esta dimensión, identifica para los demás lo que es importante (a nivel conceptual y operativo), aportando un sentido y propósito a la realidad organizativa, articulando los distintos puntos de vista. Es tarea del líder proveer dirección y guía para poner en práctica dicha visión, clarificando los procedimientos individuales para llevarla a cabo.

2. Establecer las metas. Uno de los focos centrales del liderazgo debe ser establecer las metas y propósitos centrales de la escuela.

3. Proveer estímulos intelectuales. Los líderes contribuyen a que el personal reexamine algunos de los supuestos de su práctica habitual, proveen nuevas ideas, incentivos y experiencias de desarrollo profesional. 
4. Ofrecer apoyos individualizados. Incluye la adquisición de recursos (tiempo, materiales, asesores) para el desarrollo no sólo de los miembros del personal de la escuela, sino también organizativo o institucional. El profesorado precisa sentir que la dirección les apoya, está detrás de ellos cuando surgen problemas y reduce la incertidumbre.

5. Proporcionar modelos de las mejoras prácticas, de acuerdo con los valores importantes de la organización. Proponer prácticas, como modelos ejemplares, a seguir por el personal. 6. Inducir altas expectativas de realización (resultados). Transmitir al personal altas expectativas de excelencia, consecución, calidad; de desarrollo profesional, exigir "profesionalismo" y comprometer a la escuela en el centro del cambio.

7. Construir una cultura escolar productiva. Contribuir a que el centro se configure como una cultura que promueve el aprendizaje continuo, como "comunidad profesional" donde, "desprivatizada" la práctica en el aula, se comparta el trabajo y experiencias con los colegas.

8. Desarrollar estructuras para una mayor participación en las decisiones escolares. Crear oportunidades para que todos los grupos implicados participen efectivamente en las decisiones." (1994, 57-60)

\section{Marco contextual}

En México se empezó a poner mayor atención en la gestión escolar desde la década de los 90. En 1993, se estableció el Programa de Carrera Magisterial (reformado en 1998 y 2011) que procuró elevar la calidad de la educación nacional a partir del reconocimiento e impulso a la profesionalización del magisterio y el mejoramiento de las condiciones laborales de los trabajadores de la educación. La gestión directiva se evaluó a partir de los resultados de aprendizaje de los alumnos, las actividades co-curriculares, la planeación y coordinación de trabajos con el Consejo Escolar de Participación Social, Consejo Técnico Escolar y Consejo Escolar, preparación profesional, cumplimiento a las acciones establecidas en el manual de operación relacionadas con la asesoría, y las actividades de supervisión y apoyo a los docentes. (SEP- SNTE, 1994). Aunque, el proceso parecería más una lista de cotejo, que una evaluación sobre la forma en que cada directivo llevaba a cabo su labor.

En el periodo que abarca de 1994 a 2011, se aplicaron tres programas sectoriales relacionados con la formación para la gestión de directivos (jefes de enseñanza, inspectores, supervisores, asesores técnico pedagógicos, directores y subdirectores de escuelas). (Álvarez, Topete y Abundes, 2011). Aunque, se entendía la importancia de impulsar una gestión con base en la Nueva Gerencia Publica, la formación y actuación de los directivos estaba enmarcada dentro de una administración gubernamental de tipo jerárquica y normativa, por parte de la Secretaría de Educación Pública y, además, estaba fuertemente permeada por intereses sindicales.

Con el Acuerdo Nacional para la Modernización de la Educación Básica (ANMEB), firmado en 1992, la Secretaría de Educación Pública (SEP) y el Sindicato Nacional para los Trabajadores de la Educación (SNTE), fijaron los criterios para el establecimiento del Programa Nacional para la Actualización Permanente de los Maestros de Educación Básica, el cual, fue un componente del Sistema Nacional de Formación, Actualización, Capacitación y Superación Profesional que consideraba, entre sus propósitos, el impulsar actividades de formación continua para directores.

Por su parte, el Programa de Desarrollo Educativo 1995-2000, consideró actividades de desarrollo profesional para los directores y supervisores, tanto para lo que se encontraban en servicio, como para los que accedían a esos puestos y provenían del servicio en docencia. Además, se inició una reorganización a la estructura institucional para atender directamente estas actividades. Entre las políticas que se aplicaron, se revisaron las funciones de la Dirección General de Investigación Educativa y dio lugar a la creación de la Dirección General para el Desarrollo de la Gestión e Innovación en la Subsecretaria de Educación Básica. (SEP, 2012). Esta dependencia se ha encargado de fomentar, entre otras acciones, prácticas de gestión que facilitan el alcance de los propósitos educativos.

Con la aplicación de Programas Compensatorios de Consejo Nacional para el Fomento Educativo (CONAFE), en el periodo comprendido entre los años de 1998- 2006, el cual comprendía diversos procesos de capacitación para directivos. Pero, específicamente, resalta el 
Programa para Abatir el Rezago en Educación Inicial y Básica (PAREIB), ya que, se establecieron "Redes Estatales de Asesoría para la Calidad en la Educación Primaria", con el propósito de proporcionar asesoría a los directivos para fortalecer sus habilidades de intervención y resolución de problemas en el área pedagógica y administrativa a partir de la ejecución de Proyectos de Gestión. Este proyecto, lo desarrollaba el director y servía para resolver los problemas en la calidad de la enseñanza. Además, se debía seguir métodos de trabajo con la participación de Padres de familia y la comunidad, con base en la rendición de cuentas. Como resultado en estas experiencias del CONAFE y de la Dirección General de Desarrollo de la Gestión e Innovación Educativa, desde el año 2001, la SEP inició el Programa Escuelas de Calidad y como parte de su política educativa, se desarrollaron "Estándares de Gestión para la Educación Básica para la Transformación de la Gestión Escolar y el Mejoramiento Permanente del Logro Educativo".

Para el año 2003 se implementó el Programa de Transformación de la Gestión Escolar en la Educación Básica, el cual, entre otras cosas, procuró reorientar el ejercicio de la función de los directivos escolares y fortalecer sus competencias profesionales, particularmente para que los directivos fueran capaces de lo siguiente: crear, en su zona de influencia, un ambiente de trabajo favorable para que la escuela lograra los propósitos educativos establecidos en el Plan y los programas de estudio; fortalecer los Consejos Técnicos de Zona; apoyar diferenciadamente a las escuelas de acuerdo con las necesidades detectadas en los diagnósticos de supervisión y del Centro Escolar; seleccionar los servicios y programas que respondan de manera específica a los requerimientos del Proyecto Escolar de los centros educativos de su zona; y dar seguimiento a los procesos e impacto de programas y servicios de apoyo implementados en cada plantel para mejorar la tarea y los resultados educativos. (DOF, 2003, 25 de junio). Los Estándares de Gestión para la Educación Básica para la Transformación de la Gestión Escolar y el Mejoramiento Permanente del Logro Educativo, consideraban 4 dimensiones y 20 aspectos a considerar y que daban lugar a estándares, como se muestra a continuación:

Tabla 1

Estándares de Gestión para la Educación Básica para la Transformación de la Gestión Escolar y el Mejoramiento Permanente del Logro Educativo

\begin{tabular}{|c|c|c|c|}
\hline Pedagógica Curricular & Organizativa & Particiapción social & Administrativa \\
\hline $\begin{array}{l}\text { Fomento al } \\
\text { perfeccionamiento } \\
\text { pedagógico }\end{array}$ & Liderazgo efectivo & $\begin{array}{l}\text { Funcionamiento efectivo del } \\
\text { Consejo Escolar de } \\
\text { Participación Social }\end{array}$ & $\begin{array}{l}\text { Optimización de } \\
\text { recursos }\end{array}$ \\
\hline $\begin{array}{l}\text { Planeación pedagógica } \\
\text { compartida }\end{array}$ & Clima de confianza & $\begin{array}{l}\text { Participación de los Padres de } \\
\text { familia en la escuela }\end{array}$ & Control escolar \\
\hline $\begin{array}{l}\text { Centralidad del } \\
\text { aprendizaje }\end{array}$ & Compromiso de enseñar & $\begin{array}{l}\text { Apoyo al aprendizaje en el } \\
\text { hogar }\end{array}$ & Infraestructura \\
\hline Compromiso de aprender & Decisiones compartidas & & \\
\hline \multirow{5}{*}{$\begin{array}{l}\text { Equidad en las } \\
\text { oportunidades de } \\
\text { aprendizaje }\end{array}$} & Planeación institucional & & \\
\hline & Autoevaluación & & \\
\hline & $\begin{array}{l}\text { Comunicación del } \\
\text { desempeño }\end{array}$ & & \\
\hline & Redes escolares & & \\
\hline & $\begin{array}{l}\text { Funcionamiento efectivo } \\
\text { del Consejo Técnico } \\
\text { Escolar }\end{array}$ & & \\
\hline
\end{tabular}

Fuente: SEP $(2010,73)$.

En el año 2008, con el acuerdo político denominado Alianza por la Calidad de la Educación (ACE), se establece el compromiso de construir un sistema de evaluación de la gestión escolar, entre otros aspectos. (SEP-SNTE, 2008). Con este pacto político, también se estableció la necesidad de 
contar con un sistema de estándares de desempeño y se cambiaron algunos lineamientos de Carrera Magisterial. Posteriormente, el objetivo de la gestión escolar se empezó a focalizar en los aprendizajes de los alumnos, por lo tanto, se procuró dinamizar los procesos y la participación de los actores que intervienen en la acción educativa. Esto es, el equipo de dirección de la escuela debería ser una de las instancias que debería desarrollar las competencias necesarias para la toma de decisiones, en aras de mejorar la calidad educativa e incrementar el nivel de logro de los alumnos.

Como se puede apreciar, desde un enfoque de Nueva Gestión Pública y de escuelas efectivas se procura que el directivo sea capaz de planear, organizar, controlar, administrar, evaluar con alto nivel de impacto social. Requiere la capacidad para identificar las necesidades de la escuela y su población, características del contexto, circunstancias y factores para el diseño, implementación, seguimiento y evaluación de tareas. Esto envuelve reconocer a la organización escolar en su conjunto, identificar sus elementos, la forma en que se interrelacionan y se afectan éstos entre sí. El ejercicio de tales funciones demanda del directivo habilidades complejas de pensamiento para usar el conocimiento teórico, metodológico, técnico para la ejecución de tareas específicas.

\section{La problemática detectada por la OCDE sobre la gestión escolar de México}

Desde un ámbito internacional, la Organización para la Cooperación y Desarrollo Económicos (OCDE) ha investigado, en diversos países, las características de los directivos, condiciones, actividades y su incidencia en la mejora educativa (OCDE, 2008; Pont, Nusche y Moorman, 2008). La OCDE aplica la encuesta Teaching and Learning International Survey, (TALIS, por sus siglas en inglés) que es un estudio internacional que indaga sobre la docencia, aprendizaje y dirección escolar. En el caso de México, se encontró que un porcentaje alto de los profesores mexicanos trabajan en escuelas cuyo director considera que la autonomía escolar es nula y está caracterizada por la burocracia. Los directores mexicanos reportaron que decisiones, tales como, la contratación de profesores, la fijación de salarios, el ejercicio presupuestal y la determinación de contenidos curriculares no se toman al interior de la escuela lo que les resta capacidad de actuación (OCDE, 2013).

En otro estudio, que específicamente realizó la OCDE para México, en el año 2010, se halló que el liderazgo instruccional no era una característica sobresaliente en las escuelas. Los directores no contaban con una formación, desarrollo profesional, o incentivos apropiados para enfocarse en mejorar la calidad de la instrucción, mejorar los resultados de las escuelas o consolidar los vínculos de las escuelas con la comunidad. Si bien, la normatividad institucional le ha asignado al director la función relacionada con la gestión administrativa y normativa como su principal actividad, actualmente las necesidades organizacionales requieren que tenga otro tipo de competencias que le ayuden a resolver diversos problemas de la comunidad escolar y poder alcanzar los propósitos educativos.

\section{Coherencia entre la normatividad y la práctica directiva cotidiana}

Al realizar un análisis textual sobre la forma en que se ha venido construyendo la función directiva por parte de las autoridades educativas mexicanas, en las últimas décadas, se puede ver de forma objetivada "lo deseable" en documentos, leyes y acuerdos que sustentan a la reforma educativa de 2013, pero también se pueden apreciar posibles inconsistencias de orden legal y administrativo que podrían impedir la correcta aplicación de tal reforma. Antes del año 2013, el nombramiento de los directores escolares se realizaba conforme a un sistema de escalafón, en el mejor de los casos, implementado desde 1973. Los puestos eran asignados por la Comisión Nacional Mixta de Escalafón compuesta por la Secretaría de Educación Pública (SEP) y representantes del Sindicato Nacional de Trabajadores de la Educación (SNTE). Esta comisión evaluaba a los candidatos siguiendo diversos criterios, tales como: tiempo en servicio, credenciales académicas, participación en proyectos educativos, participación en proyectos de apoyo a la comunidad, publicaciones, el diseño de material didáctico, participación en actividades de formación docente y reconocimiento previo de desempeño docente. Sin embargo, este mecanismo solo se basada en el conteo de actividades realizadas, estaba influenciado por el sindicato de docentes y 
fuertemente permeado por criterios de índole político (OCDE, 2010: 134), lo que le conferían un alto nivel de incertidumbre a la forma en que se asignaban plazas.

Con motivo de la reforma educativa del año 2013, la Secretaría de Educación Pública (SEP), en coordinación con el Instituto Nacional para la Evaluación de la Educación (INEE) llevaron a cabo una revisión de los perfiles de los directivos para el nivel de primaria y se destacó la importancia de contar con cuadros que no sólo cumplieran con tareas burocráticas, sino que también se deberían tornarse en líderes escolares y comunitarios. Esto con el propósito de que fueran un agente de cambio con capacidad de organizar a los diferentes actores, tanto al interior, como al exterior de la escuela.

A partir de la última década, se pueden identificar que las políticas educativas en México, están orientadas en la mejora de la calidad con que se presta el servicio educativo y de los aprendizajes, por lo que se implementaron los cambios necesarios en la Constitución para realizar una reforma educativa en 2013-2014 (DOF, 2013, 26 de febrero), entre otras cosas, se estableció el fortalecimiento de la gestión educativa.

En el Capítulo IV, Art. 26 a 33, se regula la promoción a cargos con funciones de dirección y de supervisión. Los cuáles solo se otorgarán por concurso de oposición, mediante convocatorias públicas en las cuales se determinarán los perfiles y requisitos. En Educación Básica, la promoción a un puesto de dirección permite obtener un nombramiento de 2 años que se considera como un periodo de inducción. Durante los mismos, el director deberá tomar cursos de desarrollo de liderazgo y gestión escolar. Al término de los dos años, puede ser promovido y se le otorga un nombramiento definitivo. En caso de no acreditar satisfactoriamente el proceso de evaluación volverá a su función docente.

Sin embargo, se puede inferir que tres aspectos entran en crisis: la forma en que desde la normatividad se propone que se ejerza la función directiva, ahora llamada gestión escolar, la transición entre normatividades, como resultado de la aplicación reciente de la reforma educativa y la práctica cotidiana del director escolar. A partir del cambio semántico, de dirección escolar a gestión escolar, se asume que el director podrá transitar hacia el cambio de identidad del puesto y sus correspondientes funciones. Por otro lado, la transición que propone la reforma de 2013 requiere de la revisión de la coherencia entre leyes, acuerdos y normas, y la creación de reglamentos en diversos niveles y ámbitos. Finalmente, se requiere que todos los agentes educativos como los propios directores, supervisores, jefes de sector y autoridades educativas apropien la normatividad y actúen en consecuencia, ya que muchos de ellos siguen laborando de acuerdo a sus sistemas de administración jerárquica y burocrática.

\footnotetext{
"Nos encontramos entonces ante dos consideraciones: por un lado, la existencia de una función directiva que, debido a las dificultades burocráticas y administrativas, no se realiza de acuerdo con las actividades que le corresponden, aunado esto a una pérdida gradual de identidad que impide a directores y supervisores reconocerse como un grupo de profesionales con competencias y conocimientos específicos para el cargo (cuidado con la afirmación de que cualquier buen maestro llegará a ser un buen director). Por otro lado, está el resurgimiento de la importancia de la tarea directiva, que destaca la necesidad de aprender nuevas actividades para generar otras tantas en el plantel escolar. Cobra importancia el hecho de plantear una reflexión cuidadosa sobre el perfil deseable del director y del supervisor, a partir de los niveles normativo y real que le otorgan características específicas a la función que ellos desempeñan.” (SEP, 2003, $110)$.
}

Con la reforma se implementaron cambios en la Constitución (DOF, 2013, 26 de febrero), se estableció que, para garantizar servicios educativos de calidad, se reestructuraría el Sistema Nacional de Evaluación Educativa y sería creado el Servicio Profesional Docente, el primero supervisa aspectos teóricos, metodológicos y técnicos de las pruebas de aprendizaje. El segundo, regula el sistema de ingreso, desempeño, promoción e incentivos a cargos de docencia, dirección, asesoría técnico pedagógica y supervisión en el nivel de la educación obligatoria. 


\subsection{Promoción a personal a cargos de dirección}

Para acceder a puestos de dirección, en el nivel de primaria, dentro del Servicio Profesional Docente (SPD), para el caso de docentes frente a grupo, solo se requiere de contar con dos años de antigüedad y presentarse a la evaluación. Para el caso de docentes que desempeñan funciones -sin plaza- de coordinación, subdirección o dirección, con o sin la categoría específica, se requiere presentarse a la evaluación, en ambas situaciones, se requiere que se oferte previamente la vacante (SEP, 2015). Se les aplican dos instrumentos para el caso de promoción: Examen de conocimientos y habilidades para la práctica profesional, Examen de habilidades intelectuales y responsabilidades ético profesionales. Para el caso de evaluación de desempeño son tres instrumentos: Informe de responsabilidades profesionales, Proyecto de Gestión Escolar del director y Examen de conocimientos curriculares y de normatividad para la función de dirección. Si bien, este proceso hace más transparente la selección de personal para puestos directivos, es necesario considerar los conocimientos o experiencia que se requieren para acceder a la plaza de director.

La Ley General del Servicio Profesional Docente, establece para los docentes frente a grupo, que puedan acceder al puesto, contando con dos años de antigüedad y para las otras figuras solo se debe contar con el desempeño extraoficial en el cargo. Evidentemente, se esta excluyendo del proceso a candidatos externos al sistema educativo de sostenimiento Estatal. Esta forma de reclutamiento y selección requiere de una revisión, ya que el perfil de puesto, debería establecer una formación específica en áreas relacionadas con la administración educativa y pedagogía o áreas de conocimiento similares y un nivel mínimo de experiencia en el puesto. Si bien, una vez, que se obtiene el puesto, se trata de subsanar las áreas de oportunidad, mediante cursos, esto no resulta ser un proceso ideal para la formación en gestión escolar, ya que va en detrimento de la calidad educativa.

\subsection{Perfil de desempeño directivo}

Ahora bien, en cuanto a las funciones, la Secretaría de Educación Pública diseñó los Perfiles, parámetros e indicadores (PPI) para la evaluación a promoción a cargos con funciones de dirección y evaluación de desempeño (SEP, 2016). El diseño de estándares se basó en las experiencias pasadas y los trabajos realizados en otros países. Este documento orienta las características de las tareas y la forma en que se espera el director que se desempeñe en la función. Los Perfiles, parámetros e indicadores (PPI) se componen de cinco áreas de desarrollo o ámbitos de acción directivo llamadas dimensiones y comprenden un nivel individual, escolar y comunitario.

En el PPI, la dimensión que orienta las funciones de liderazgo se pueden ubicar en la 2 y 5 . Establecen lo que se espera que desarrolle el director con respecto a su actividad de liderazgo en la escuela y la comunidad. La dimensión 2, establece que el director debe ejercer una gestión escolar eficaz para la mejora del trabajo en el aula y los resultados educativos de la escuela. Para tal efecto, debe realizar acciones para organizar el trabajo de la escuela y mejorar los resultados educativos. Además, implementar acciones para la mejora escolar y la calidad de los aprendizajes de los alumnos; gestionar la mejora de las prácticas docentes y el logro de los aprendizajes en los alumnos, gestiona los recursos, espacios físicos y materiales para el funcionamiento de la escuela y capacidad para construir ambientes de trabajo en la escuela donde sea posible que todos aprendan. Por su parte, la dimensión 5, determina que el director debe reconocer el contexto social y cultural de la escuela y establecer relaciones de colaboración con la comunidad, la zona escolar y otras instancias, para enriquecer la tarea educativa. Para lo cual, debe considerar la diversidad cultural y lingüística de la comunidad y su vínculo con la tarea educativa de la escuela; gestionar la colaboración de las familias, de la comunidad y de otras instituciones en la tarea educativa de la escuela, y aplicar estrategias para el funcionamiento eficaz con otras escuelas y el trabajo con otros directivos.

Sin embargo, se requiere de las otras dimensiones para poder cumplir con una gestión escolar eficaz para construir ambientes de trabajo en la escuela donde sea posible que todos aprenda requerirá gestionar la colaboración de autoridades educativas, docentes, personal administrativo, 
operativo, Padres de Familia, comunidad, otras escuelas y de otras instituciones diferentes a la educativa de la escuela. La dimensión 1, considera que el directivo debe tener la capacidad para explicar: la tarea fundamental de la escuela; los rasgos de la organización y el funcionamiento de una escuela eficaz; los componentes del currículo y su relación con el aprendizaje de los alumnos, y los elementos del trabajo en el aula y las prácticas docentes. La dimensión 3, aborda la capacidad directiva para reflexionar sistemáticamente sobre su práctica profesional como medio para mejorarla; emplear estrategias de estudio y de aprendizaje para su desarrollo profesional y utilizar diferentes medios para enriquecer su desarrollo profesional. La dimensión 4, se enfoca en los conocimientos que debe tener en cuanto a los principios filosóficos, fundamentos legales y finalidades de la educación pública mexicana; la capacidad para gestionar ambientes favorables para el aprendizaje, la sana convivencia y la inclusión educativa e implementar acciones para mantener la integridad y seguridad de los alumnos en el aula y en la escuela.

Se sobreentiende que, a partir de estas dimensiones, se está adaptando la función directiva en el trabajo cotidiano, lo cual, no es necesariamente correcto, debido al poco tiempo de implementación de la reforma (2013); las presiones burocráticas que no terminan de asimilar las nuevas funciones de acuerdo con los estándares de evaluación, la falta de coherencia entre leyes, acuerdos y normas, y el ajuste que se realiza en cada escuela de tales políticas, considerando factores contextuales de tipo administrativo, social, político, sindical, económico, etcétera .

Si bien, el modelo que propone la SEP está en consonancia con otros marcos teóricos y modelos que se impulsan para mejorar las prácticas de gestión para elevar la calidad del servicio, como el propuesto por Leithwood (1994, p. 57-60). Las acciones que desarrollan los directivos escolares mexicanos están fuertemente influenciadas por marcos reguladores y de control que sobre-enfatizan los procesos burocráticos y políticos. Esto quizá es debido a que la gestión escolar mexicana, tradicionalmente se ha basado en el seguimiento a la normatividad, lo que limita la capacidad de gestión, autonomía e innovación.

En primer lugar, se requiere hacer una revisión de la coherencia entre normatividades en los diferentes niveles. Esto para propiciar la gobernanza regulatoria multi-nivel. Una mejor regulación en todos los niveles de gobierno, evitaría la falta de coordinación, superposición o falta de claridad en las funciones y responsabilidades. Un ejemplo de lo anterior es la normatividad que se establece en los Acuerdos 98, 96 y 97 que aluden al funcionamiento de los centros escolares en los niveles de primaria y secundaria. Tales Acuerdos fueron ratificados con el Acuerdo número 01/03/14 dentro del marco de la Reforma Educativa de 2013 (DOF, 2014b, enero 3). El Acuerdo número 96, que establece la organización y funcionamiento de las escuelas primarias establece: "Entre las funciones principales del personal directivo está el "administrar en el plantel a su cargo la prestación del servicio educativo del nivel primaria, conforme a las normas y los lineamientos establecidos por la Secretaría" (DOF, 1982, 7 de diciembre: Capitulo IV, Articulo 14).

Estos acuerdos puntualmente determinan cada una de las actividades que se espera que realice el director y son básicamente funciones administrativas, lo que da lugar a la focalización de evidencias desde las que, cotidianamente, son evaluados los directivos por sus superiores. Al solo considerar que se debe dar seguimiento a la normatividad, propone actividades de tipo administrativo que no tiene el sentido de los Perfiles, parámetros e indicadores.

En este sentido, Ornelas (2008) evidenció que los directores no disponían de un recurso económico adecuado para enfrentar algunos problemas de la escuela, tenían autoridad, pero no poder para hacer que los maestros acudieran a dar sus clases, cumplieran con los programas oficiales y realizaran la evaluación del alumnado. Además, debían tener tiempo y hacer que funcionaran: el Consejo Técnico Escolar, el Consejo Escolar de Participación Social, Consejo Técnico de Zona, así como, la Asociación de Padres de Familia. También, debían representar a la escuela ante las autoridades superiores; evaluar el desempeño de sus docentes y actuar en consecuencia; atender el llenado de diversos formularios, de los cuales llegó a contar cerca de 100, a veces con los mismos datos solicitados, pero, en un formato distinto para diversas dependencias. Esto, sin dejar de considerar que era responsable de la infraestructura, de ordenar las tareas de mantenimiento, 
limpieza de las escuelas y atención de Padres de familia, entre lo más relevante.

Con la evaluación que implica ingresar al SPD, se estableció el fortalecimiento de la gestión educativa mediante el apoyo permanente de autoridades educativas de distintos niveles de gobierno. En el Acuerdo secretarial 717 se establece para las escuelas:

"Para fortalecer su autonomía de gestión, requieren de la atención permanente de las autoridades educativas locales y municipales; del liderazgo del director; del trabajo colegiado del colectivo docente; de la supervisión permanente de los procesos de enseñanza y de aprendizaje que se producen en las aulas; de la asesoría y apoyo para el desarrollo escolar y del involucramiento de los padres de familia y de la comunidad en general para que de manera colaborativa participen en la toma de decisiones y se corresponsabilicen de los logros educativos.” (DOF, 2014a, 7 de marzo: s/p).

Además, establece que el director debe garantizar una administración transparente y eficaz de los recursos financieros que reciba y en su actividad, para lo cual, estará acompañado por el Consejo de Participación Ciudadana y el Consejo Escolar (SEP, 2003; DOF, 2014a, 7 de marzo). Lo anterior no está en concordancia con lo que se espera del desempeño proactivo de los directivos, ya que depende de una figura organizacional llamada Servicio de Asistencia Técnica a la Escuela en la Educación Básica (SATE) ${ }^{2}$, el cual deberá asegurar la participación activa de todos los involucrados en el proceso educativo. Supone un cambio en la forma de entender la administración de la organización de los planteles educativos, ya que se pasa de una administración tipo jerárquica a una horizontal y con una suerte de "staff de apoyo". Se apuesta a que el liderazgo eficiente de los directivos, el trabajo colegiado entre docentes y el apoyo técnico pedagógico de los supervisores resulten en la mejora de la calidad educativa, con una perspectiva de inclusión y equidad (SEP, 2017). Pretender aplicar una política de "escuela al centro", implica que el director identifique necesidades o problemas específicos y otros agentes educativos o gubernamentales apoyen para la satisfacción o solución de esos problemas, rompiendo el orden jerárquico que implica que el Supervisor, Jefe de sector u otras autoridades educativas apoyen, actúen o asesoren en función de lo que ellos consideren o les indiquen. Actualmente, esto podría resultar poco aceptado por aquellos que, dentro del organigrama, son los superiores y los que debieran dirigir la administración educativa.

Entre los aspectos que se pueden identificar como problemáticos están los siguientes: a) Es común que se sature a la escuela con la aplicación de múltiples proyectos, programas o trámites que requieren ser atendidos por el director lo cual le demanda tiempo; b) Los supervisores de los directores con base en el cumplimiento a la norma administrativa de los Acuerdos 96, 97 y 98 buscan únicamente evidencias de trabajo de control administrativo o contable; c) Los directores tienen capacidad limitada para tomar decisiones sobre el manejo de recursos humanos, económicos, administrativos o técnico-pedagógicos ${ }^{3}$; d) No cuentan con atribuciones legales en materia laboral sobre su personal (requiere una figura jurídica y administrativa que le permita tomar decisiones de alto impacto con respecto a su personal sindicalizado o eventual; e) La SEP y las Escuelas Normales requieren implementar programas de formación específica para directores, el ser docente no basta para ejercer la función ${ }^{4}$; f) La evaluación de promoción y desempeño con que la

\footnotetext{
${ }^{2}$ En algunos estados, el Servicio de Asistencia Técnica a la Escuela en la Educación Básica se ha incluido en el organigrama de las Secretarías de Educación Pública estatal, como direcciones, como instancias dependientes de otras direcciones o como equipos de trabajo compuestos por Jefes de Sector, Supervisores Escolares, Asesores técnicopedagógicos, Jefes de Enseñanza o Directores Técnicos, pero en la mayoría de estados su implementación aún se encuentra en ciernes. Quizá el problema se debe a que el SATE se compone de la figura de Asesor Técnico Pedagógico (ATP), de Lenguaje oral y escrito, y pensamiento matemáticas. Su contratación o regularización de plaza se ha complicado en algunos estados, ya que no tienen recursos para la contratación; no existe la clave presupuestal para la formalización del puesto que ya ocupan en esa función docentes "comisionados"; se presentaron problemas técnicos en los instrumentos de evaluación del concurso de oposición, por lo que se retrasó el proceso de contratación a nivel nacional y existe falta de claridad en las funciones.

3 Aunque se acepta cierta libertad para adecuar el currículo a las necesidades de la escuela o estado, por parte de la Secretaria de Educación Pública federal, se debe cumplir con la enseñanza de todos los contenidos de los programas, debido a que las evaluaciones nacionales de aprendizaje no consideran modificaciones particulares en la enseñanza de los mismos.

${ }^{4}$ La Universidad Pedagógica Nacional cuenta con la carrera en Administración Educativa, pero la mayoría de los
} 
actualmente se evalúa a directores, se basa en instrumentos de muy corto alcance, que no dan cuenta de las exigencias y complejidad del trabajo que se enfrentará como directivo; g) Los criterios para acceder al puesto deben considerar experiencia y formación específica en administración y áreas vinculadas a la educación; h) se debe realizar una revisión entre leyes, acuerdos, normas y formas de trabajo cotidiano para hacerlos coherentes; i) los sistemas de evaluación basados en exámenes de conocimiento y elaboración de proyectos, requiere de sistemas de evaluación y productos más complejos para dar cuenta de las conductas que implican habilidades cognitivas y no cognitivas, finalmente, j) Se debe dar la posibilidad de participar en los concursos de oposición a candidatos externos al sistema educativo gubernamental, que cuenten con las credenciales académicas y experiencia.

\section{Conclusiones}

Actualmente, se reconoce que la función directiva es una pieza clave para elevar la calidad de aprendizajes en las escuelas. Por lo cual, dicha función, se ha tornado en una prioridad de la política pública educativa nacional. Tomando como base, los conocimientos sobre la gerencia y el liderazgo que se han desarrollado en empresas privadas se procura directores más eficientes y eficaces para las escuelas. Se entiende que las escuelas son organizaciones básicas que deben tener la capacidad para responder a los retos educativos a nivel nacional y local. El trabajo que realizan los directores en sus centros escolares debe incidir de manera directa sobre el comportamiento de los alumnos, docentes y demás actores que participan en la organización del plantel y, en su conjunto, generar un clima escolar propicio para el aprendizaje. Por tal razón, desde hace décadas el gobierno mexicano ha implementado diversas políticas y programas que se enfocan en mejorar el desempeño del director.

Una forma de entender la función directiva se puede apreciar con la aplicación de la reforma de 2013. Los perfiles parámetros e indicadores que se establecen para la promoción a cargos con funciones de dirección de la SEP, considera diversos aspectos sobre la profesionalización a la función directiva desde un marco de escuelas efectiva y la Nueva Gerencia Pública. Sin embargo, las acciones que desarrollan los directivos escolares mexicanos están fuertemente influenciadas por marcos reguladores y de control que sobre-enfatizan los procesos administrativos y con una organización de tipo jerárquico. Esto quizá es debido a que la gestión escolar mexicana, tradicionalmente se ha basado en el seguimiento a la normatividad, lo que ha servido para guiar las actividades cotidianas del directivo y tener control sobre sus actividades, lo que limita su capacidad de gestión, autonomía y capacidad de innovación.

En primer lugar, se observó que existen inconsistencias o por lo menos, falta entender el espíritu de las leyes de la reforma de 2013, para hacer los correspondientes ajustes y propiciar una gobernanza regulatoria multi-nivel. Esto es, se debe ajustar la normatividad y procesos institucionales para evitar que se siga trabajando bajo sistemas legales, administrativos, sindicales de tipo burocrático que no responden a los Perfiles, parámetros e indicadores esperados y, por ende, afecta la evaluación de desempeño directivo de la SEP. En segundo lugar, el proceso de promoción a cargos de dirección, sigue siendo deficiente debido a los criterios establecidos para acceder al puesto, lo que no garantiza una formación adecuada o la experiencia necesaria para el puesto. Además, es necesario abrir el concurso a interesados que no pertenecen al sistema educativo de financiamiento Estatal, pero que cuentan con la experiencia y credenciales académicas necesarias. En tercer lugar, se puede inferir que los instrumentos que se usan para la evaluación para promoción o desempeño directivo, si bien pueden dar cuenta de los conocimientos de los directores, en diversas áreas o de su capacidad de realizar un proyecto de mejora, aún requiere de instrumentos más sofisticados para dar cuenta de aquellas habilidades cognitivas y no cognitivas. En cuarto lugar, debido a las prácticas sindicales e institucionales, el director no tiene un control total de sus trabajadores, por lo que no puede ejercer un liderazgo o dirección adecuada, se debe dotar a los directores de autoridad legal para tomar las medidas correspondientes con respecto al desempeño de su personal. En quinto lugar, se pretende que con la política de "escuelas al centro" y la creación del SATE, todos los actores sociales y educativos actúen en función de las necesidades escolares, lo 
que implica una organización de tipo horizontal y con un staff, situación que no necesariamente es congruente con la administración de tipo jerárquica y burocrática de la administración gubernamental actual. Finalmente, debido a la complejidad del trabajo la Secretaría de Educación Pública debe configurar un sistema de formación directiva o programas que permitan a los docentes interesados en ser promovidos obtener las competencias necesarias antes de concursar por la vacante. Además, de abrir la convocatoria de ingreso, al puesto directivo, a candidatos que tienen la formación en pedagogía o áreas afines y se han formado en administración de educativa a nivel de licenciatura o posgrado y con experiencia. Así, es importante considerar que, al parecer, la evaluación e ingreso y desempeño que se requiere para ingresar al Servicio Profesional Docente presenta una brecha con respecto a lo que considera que un directivo debe hacer con lo que realmente sucede en vida cotidiana de la escuela.

\section{Referencias}

Álvarez, E., \& Pérez R. (2011). Liderazgo directivo en los centros educativos de Asturias. Bordón, Revista de Pedagogía, 63(3), 23-4.

Álvarez, I., Topete, C., \& Abundes, A. (2011). El concepto emergente de gestión educativa estratégica y desafíos para la formación en gestión, Ponencia. XI Congreso Nacional de Investigación Educativa. México.

Cohn, E., \& Rossmiller, R.A. (1987). Research on effective schools: implications for less developed countries. Comparative Education Review, 31(3), pp. 377-399. doi: 10.1086/446697.

Bass B., \& Avolio B. (Eds.) (1990). Improving organizational effectiveness through transformational leadership. Thousand Oaks, GA: Sage.

Branch, G., Hanushek, E., \& Rivkin, S. (2013). School Leaders Matter: Measuring the Impact of Effective Principals. Education Next, 13(1), pp. 63-69.

Burns, J. (1978). Leadership. New York: Free Press.

Bush, T., \& D. Glover (2004). Leadership Development: Evidence and Beliefs. Nottingham, UK: National College for School Leadership.

DOF (1982, 7 de diciembre) Acuerdo secretarial 98. Sobre La Organización Y Funcionamiento de las Escuelas De Educación. Recuperado el día 3 de octubre de 2017 de: https://www2.sepdf.gob.mx/convivencia/conoce_marco/archivos/acuerdo96.pdf.

DOF (1982, 7 de diciembre). Acuerdo Número 96. Que Establece la Organización y Funcionamiento de las Escuelas Primarias. Recuperado el día 3 de octubre de 2017 de: https://sites.google.com/site/normatividadsep/acuerdo-96

DOF (2003, 25 de junio), Reglas de Operación del Programa de Transformación de la Gestión Escolar en la Educación Básica, 2003 [Documento electrónico]. Función Pública. Recuperado el día 30 de abril de 2015 de:

http://www.funcionpublica.gob.mx/scagp/dgorcs/reglas/2008/11\%20SEP\%2008/1111\%20SEP\%2003\%20P.\%20Transf.\%20G.E.\%20E.B.\%20ROP\%20250603.doc.

DOF (2013, 26 de febrero), Decreto por el que se reforman los artículos 30 . en sus fracciones III, VII y VIII;y 73, fracción XXV, y se adiciona un párrafo tercero, un inciso d) al párrafo segundo de la fracción II y una fracción IX al artículo 3o. de la Constitución Política de los Estados Unidos Mexicanos. Secretaría de Gobernación. Recuperado el día 14 de julio de 2017 de: http://www.dof.gob.mx/nota_detalle.php?codigo $=5288919 \&$ fecha $=26 / 02 / 2013$.

DOF (2014a, 7 de marzo). Acuerdo secretarial número 717. Lineamientos para formular los Programas de Gestión Escolar (documento electrónico). Recuperado el día 14 de julio de 2017 de: http://estudiaen.jalisco.gob.mx/cepse/sites/estudiaen.jalisco.gob.mx.cepse/files/acuerdo_717_ programa_de_gestion_escolar.pdf.

DOF (2014b, enero 3). Acuerdo número 01/03/14 por el que se abrogan los acuerdos secretariales que en el mismo se indican, en virtud de que su objeto se ha cumplido; se dan a conocer los que ban quedado sin efectos, por disposición expresa o por tener establecida una vigencia temporal, así como los que continúan vigentes para el sector educativo. Recuperado el día 3 de octubre de 2017 de: http://www.dof.gob.mx/nota_detalle.php?codigo $=5338451 \&$ fecha $=27 / 03 / 2014$.

Levine, D., \& Lezotte, L. (1990). Unusually effective schools: a review and analysis of research and practice. Madison: National Center for Effective Schools Research and Development.

Leithwood, K. (1994). Liderazgo para la reestructuración de las escuelas. Revista de Educación, 304, 
pp. 31-60. doi: 10.1007/978-94-009-1573-2_23.

Leithwood, K., Tomlinson, D., \& Genge, M. (1996), Transformational school leadership. En Leithwood, K.A., Chapman, J.D., Corson, P., Hallinger, P., \& Hart, A. (Eds.) International Handbook of Educational Leadership and Administration (pp. 785-840). New York: Springer.

Losada, C. (1999). ¿De Burócratas a Gerentes? Washington: Banco Interamericano de Desarrollo.

Mortimore, P. (1991). The nature and findings of school effectivesness research in the primary sector. En Riddell, S., \& Brown, S. (Eds.) School Effectiveness Research: Its Messages for School Improvement. Edinburgh: HMSO.

Muhammad Faizal A. G. (2008). Pembentukan Model Keberkesanan dan Penambabbaikan Sekolah. Unpublished PhD dissertation. University of Malaya.

OCDE (2010). Mejorar las escuelas: estrategias para la acción en México. Paris: OECD Publishing.

OECD (2008). Improving School Leadership Volume 1: Policy and Practice. Paris: OECD Publishing.

OECD (2013). Teaching and Learning International Survey TALIS 2013 Conceptual Framework. Otawa: IEA.

Ornelas, C. (1995). El sistema educativo mexicano. La transición de fin de siglo. México D.F.: FCE.

Ornelas, C. (2008). Política, Poder y Pupitres: Crítica al Nuevo Federalismo Educativo. México D.F.: Siglo XXI.

Paul, S. (1983). Training for Public Administration and Management in Developing Countries. A Review. World Bank Staff. Working papers num. 584. Management and development series number 11 [documento electrónico]. Recuperado el día 7 de mayo de 2015 de: http://wwwwds.worldbank.org/external/default/WDSContentServer/WDSP/IB/1999/09/17/000178830 _98101903415942/Rendered/PDF/multi_page.pdf.

Pollit, C., \& Bouckaert, G. (2000). Public Management Reform: A Comparative Analysis. Oxford: Oxford University Press.

Pont, B., Nusche, D., \& Moorman, H. (2008). Mejorar el liderazgo escolar. Volumen 1: política y práctica. Paris: OECD Publishing.

Pozner, P. (1997). El directivo como gestor de aprendizajes escolares. Buenos Aires: AIQUE.

SEP-SNTE (1994). Carrera Magisterial, Prontuario, 2da vertiente. México D.F.: SEP.

SEP-SNTE (2008). Alianza por la Calidad de la Educación entre el gobierno Federal y los Maestros de México representados por el Sindicato Nacional de trabajadores de la Educación. México D.F.: SNTE.

SEP (2003). Antologia: Gestión Escolar. Programa Nacional de Carrera Magisterial. México D.F.: SEP.

SEP (2010). PETE Simplificado. Recomendaciones para elaborar el Plan Estratégico de Transformación Escolar. México: SEP.

SEP (2012). Manual de organización de la Dirección General de Desarrollo de la Gestión e Innovación Educativa. México: SEP.

SEP (2015). Programa de Promoción en la Función por Incentivos en Educación Básica. México D.F.: SEP. [Documento electrónico]. Recuperado el día 20 de octubre de 2017 de: http://servicioprofesionaldocente.sep.gob.mx/2016/docs/PFI.pdf.

SEP (2016). Perfiles, parámetros e indicadores para el ingreso a funciones directivas. Concursos de oposición para las Promociones a cargos con funciones de Dirección y de Supervisión, y a las Funciones de Asesoría Técnica Pedagógica en Educación Básica Ciclo Escolar 2017-2018. Recuperado el día 14 de julio de 2017 de: http://servicioprofesionaldocente.sep.gob.mx/content/ba/docs/2017/ingreso/PPI_INGRES O_EB_2017_2018.pdf.

SEP (2017). Lineamientos generales para la prestación del Servicio de Asistencia Técnica a la Escuela en la Educación Básica. Recuperado el día 29 de enero de 2018 de: http://servicioprofesionaldocente.sep.gob.mx/content/general/docs/2017/LINEAMIENTOS _SATE.pdf.

Sharifah M., \& Lewin, K. (1993). Insights into science education: Planning and policies in Malaysia. Paris: UNESCO. 
